\title{
Exciton and Carrier Dynamics in Two-Dimensional Perovskites
}

Andrés Burgos-Caminal, Etienne Socie, Marine E. F. Bouduban, and Jacques-E. Moser*

Photochemical Dynamics Group, Institute of Chemical Sciences and Engineering, and Lausanne Centre for Ultrafast Science (LACUS),

École Polytechnique Fédérale de Lausanne, 1015 Lausanne, Switzerland

* Corresponding author, email: je.moser@epfl.ch

\section{Supporting Information}

\section{S1. Main results}

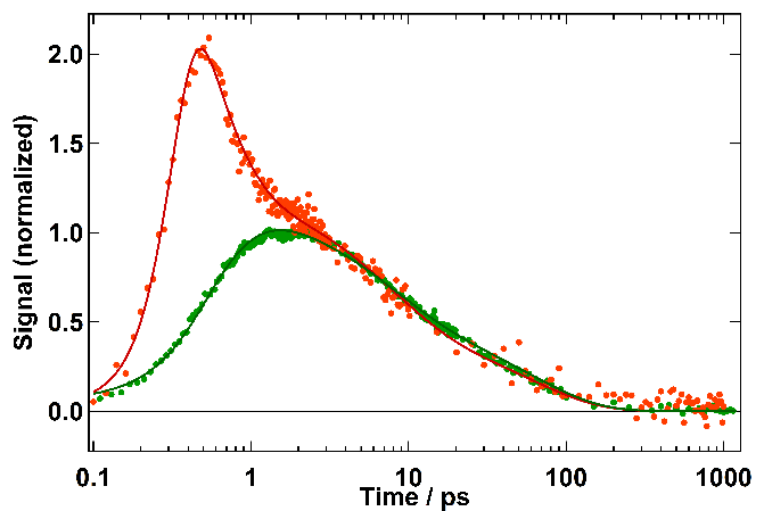

Figure S1. Full view of the decay in Figure 1A with a different normalization to highlight the coinciding decays.

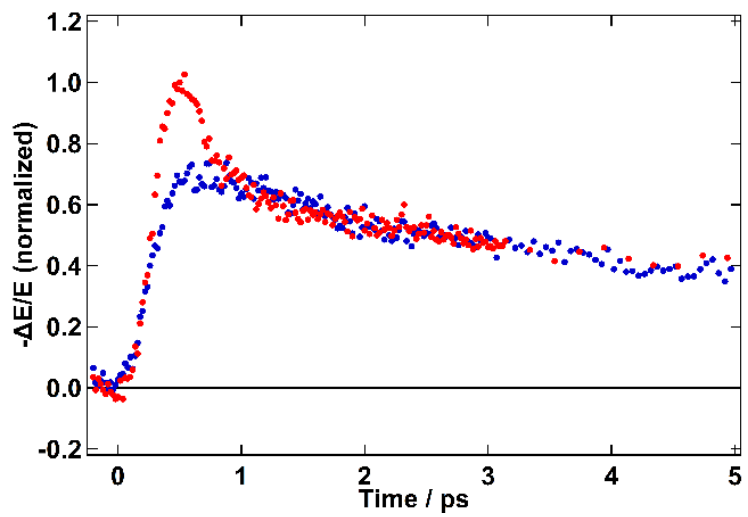

Figure S2. Figure 1B with a different normalization to highlight that the main difference lies in the initial decay. 

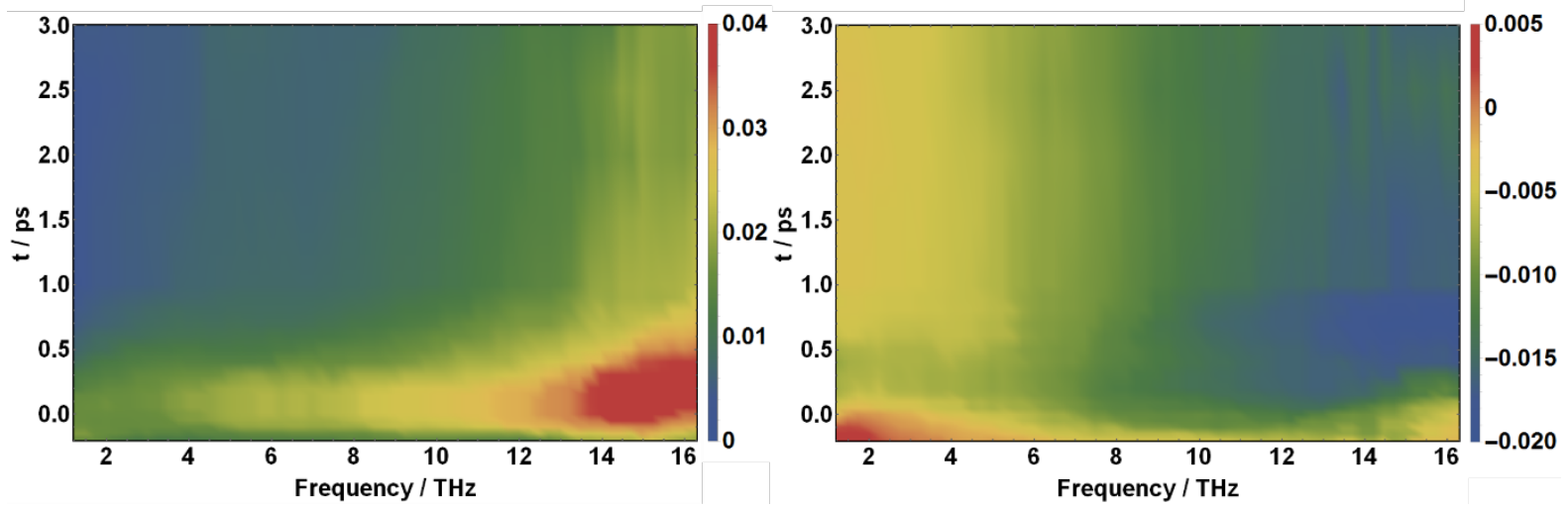

Figure S3. 2D maps of the real (left) and imaginary (right) parts of the $-\Delta E / E$ signal corresponding to the data in Figure 2. The maps have been cleaned with a median filter.

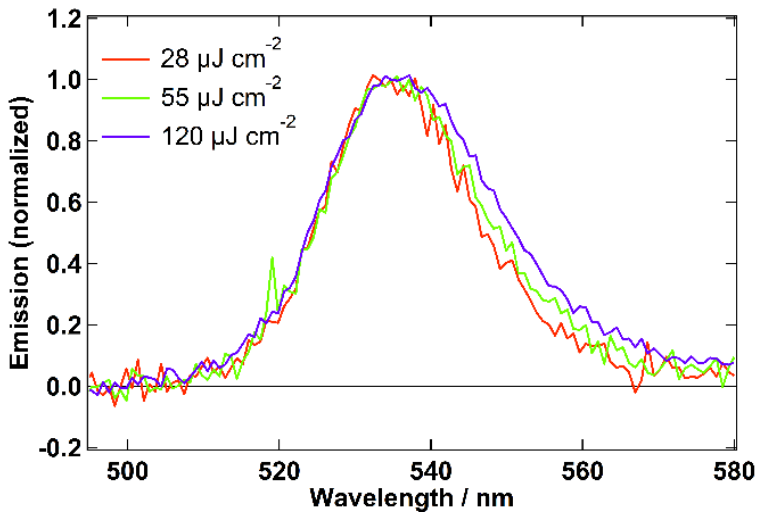

Figure S4. Normalized emission obtained from averaging the 0.96-1.04 ps range for different fluences. At higher fluence, additional contributions are only seen on the low energy side of the band. 

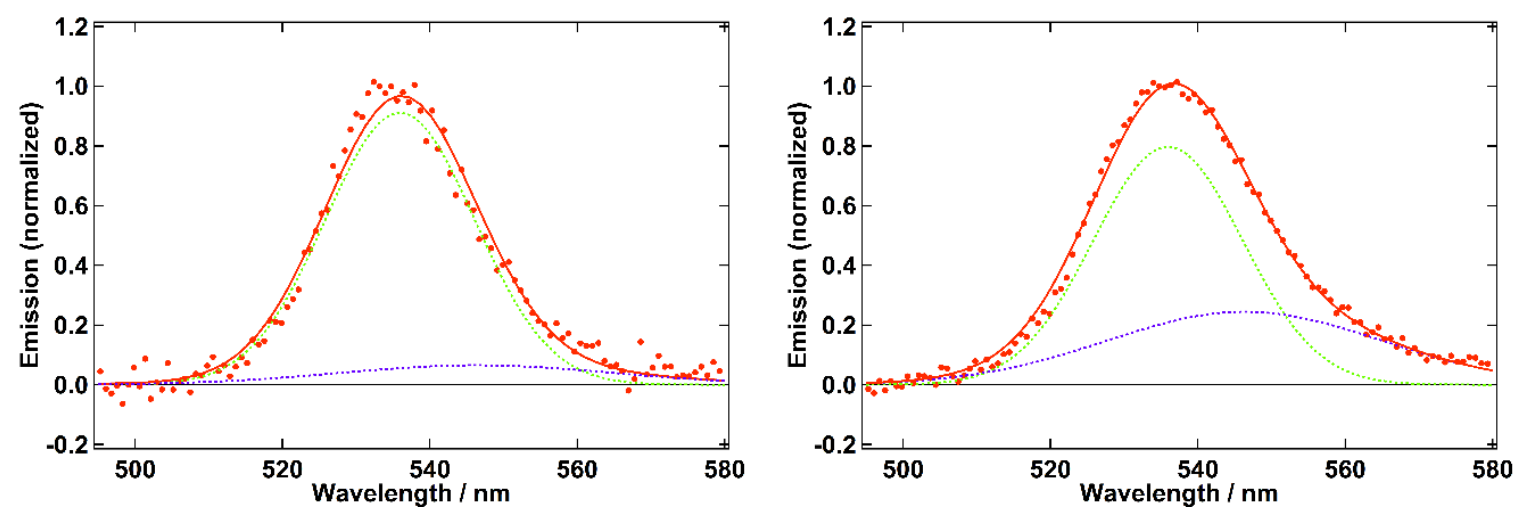

Figure S5. Multipeak fit of the emission at 1 ps for $28 \mu \mathrm{J} \mathrm{cm} \mathrm{cm}^{-2}$ (left) and $120 \mu \mathrm{J}$ $\mathrm{cm}^{-2}$ (right). The main peak is centered at $536 \mathrm{~nm}$ while the second peak is the result of a red shift of $44 \mathrm{meV}$. The second peak is clearly more important at a higher fluence. The widths and positions are equal for the two fits.
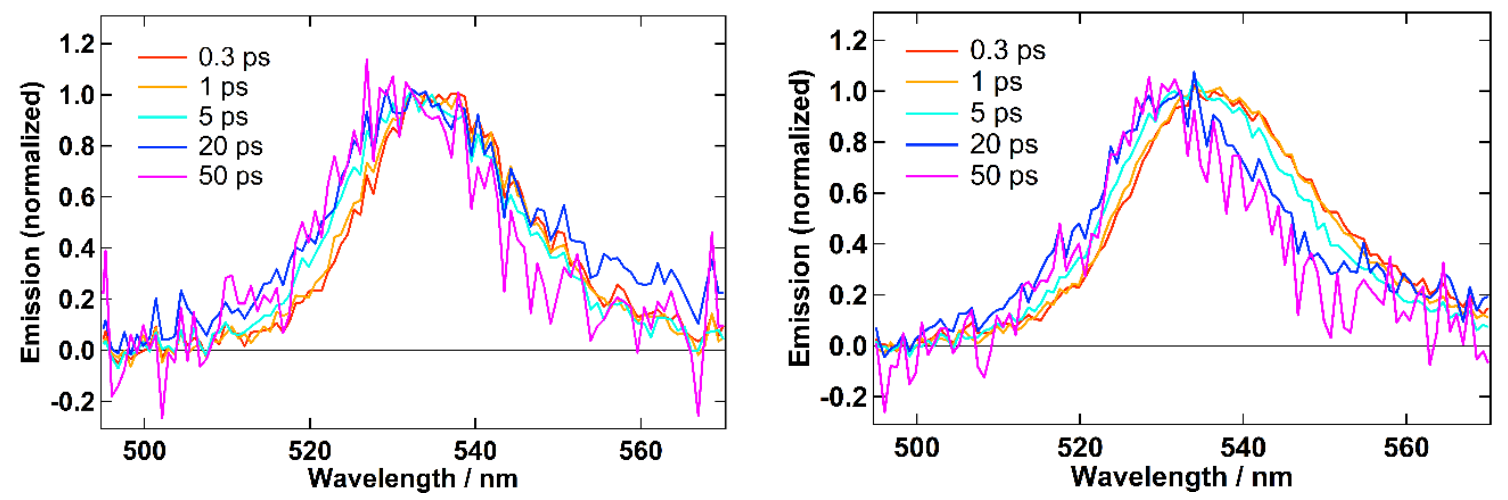

Figure S6. Normalized emission at $28 \mu \mathrm{J} \mathrm{cm}^{-2}$ (left) and $120 \mu \mathrm{J} \mathrm{cm}^{-2}$ (right) for different time delays. There is an appreciable blue shift that is not fluence dependent. The biexcitonic contribution on the red side is clearly dependent on the fluence and follows different kinetics. 
Table S1. Fluence dependence of the tri-exponential fit for the $-\Delta E / E$ decay at $\lambda_{\mathrm{ex}}=400 \mathrm{~nm}$. The first time constant can be related to carrier cooling while the latter two can be assigned to recombination.

\begin{tabular}{|l|l|l|l|}
\hline$F / \mu \mathrm{Jcm}^{-2}$ & $\tau_{1} / \mathrm{ps}$ & $\tau_{2} / \mathrm{ps}$ & $\tau_{3} / \mathrm{ps}$ \\
\hline 6.2 & $0.29 \pm 0.03$ & $12.5 \pm 2.2$ & $360 \pm 35$ \\
\hline 19 & $0.29 \pm 0.02$ & $9.8 \pm 1.1$ & $248 \pm 36$ \\
\hline 37 & $0.31 \pm 0.02$ & $8.5 \pm 0.8$ & $198 \pm 35$ \\
\hline 56 & $0.29 \pm 0.02$ & $7.1 \pm 0.6$ & $111 \pm 21$ \\
\hline 80 & $0.33 \pm 0.02$ & $6.1 \pm 0.5$ & $92 \pm 16$ \\
\hline
\end{tabular}

\section{S2. TRTS data treatment}

In order to obtain the data reported in Figure $2 \mathrm{~A}$, we need to calculate $\Delta \sigma(\omega)$. For that, we can use the following equation ${ }^{1}$

$$
\Delta \sigma(\omega)=-\frac{\varepsilon_{0} c\left(n_{A}+n_{B}\right)}{L} \frac{\Delta E(\omega)}{E(\omega)}
$$

where $n_{A}=1.00$ and $n_{B}=1.54$ are the refractive index of the air and of the HDPE substrate, $\varepsilon_{0}$ is the vacuum permittivity, $c$ is the speed of light, $L=550 \mathrm{~nm}$ is the thickness of the photoactive layer (Figure S11), and $\Delta E$ and $E$ are the Fourier transforms of difference in THz electric field upon photoexcitation and the original one, respectively. In the text, we often refer to $-\Delta E / E$ as the $\mathrm{THz}$ absorption signal. This equation is valid for thin films assuming that $\Delta E$ is small compared to $E$. In addition, this equation is valid under a steadystate condition, where $\Delta \sigma(\omega)$ does not substantially change during the duration of the pulse. However, this is not always the case, especially at very short pump-probe delays. A solution was introduced by Beard et al. where a 2D map is obtained and $\Delta \sigma(\omega, \tau)$ is obtained along the diagonal. ${ }^{2}$ Likewise, $\Delta E$ can directly be measured scanning simultaneously the pump-probe and the gate-probe delays, allowing to obtain $\Delta E$ waveforms where all the data points have a constant delay $(\tau)$ from the arrival of the pump. $^{3}$ 


\section{S3. Kinetic model}

The kinetic model presented in this work is based on following the densities of excitons $\left(N_{e x}\right)$, hot carriers $\left(N_{h c}\right)$, and cold carriers $\left(N_{c c}\right)$ over time $(t)$. Thus, we define three rate equations :

$$
\begin{gathered}
\frac{d N_{e x}(t)}{d t}=a(G(t))_{510 n m}+k_{e f} N_{c c}(t)^{2}-k_{e r} N_{e x}(t)-k_{a} N_{e x}(t)^{2}-k_{e d} N_{e x}(t), \\
\frac{d N_{h c}(t)}{d t}=a(G(t))_{400 n m}+k_{a} N_{e x}(t)^{2}-k_{c} N_{h c}(t), \\
\frac{d N_{c c}(t)}{d t}=-2 k_{e f} N_{c c}(t)^{2}+k_{c} N_{h c}(t)+2 k_{e d} N_{e x}(t)
\end{gathered}
$$

where $k_{e f}, k_{e r}, k_{a}, k_{e d}$, and $k_{c}$ are the kinetic constants for exciton formation, exciton recombination, exciton-exciton annihilation, exciton dissociation, and carrier cooling, respectively. In addition, $G(t)$ is the generation term defined as :

$$
G(t)=\sqrt{\frac{4 \log (2)}{\pi w^{2}}} \exp \left(-\frac{4 \log (2)\left(\mathrm{t}-\mathrm{t}_{0}\right)^{2}}{w^{2}}\right),
$$

where $w$ is the fwhm of the Gaussian. A term "2" is used to indicate that two carriers are taken or generated when one exciton is formed or dissociated. Alternatively, no such term is used for the Auger process since two excitons turn into two hot carriers. Furthermore, $k_{e d}$ is considered to be effectively zero, due to the large $E_{b}(270 \mathrm{meV})$. We can approximately consider that $k_{e d}=k_{e f} / \exp \left(-\frac{E_{b}}{k_{b} T}\right)=k_{e f} / 4.4 \cdot 10^{4} \approx 0$.

The signal is expressed as $S=N_{c c}+c_{h c} N_{h c}+c_{e x} N_{e x}$, where $c_{e x}$ and $c_{h c}$ are the proportionality coefficients for excitons and hot carriers, respectively. $a$ is the amplitude coefficient in density units. It serves as a measure of the fluence. During the global fit, only one value of $a$ is fitted for each wavelength. The different values for each fluence are obtained multiplying that value by a coefficient $b=F_{i} / F_{0}$ where $F_{i}$ is the fluence used in that particular trace and $F_{0}$ is the lowest fluence used.

The equations are solved numerically and fitted using a home-written code in Wolfram Mathematica. The results of the fit are shown in Table S2. 
Table S2. Results of the global fit with their corresponding fit error, where "adu" stands for arbitrary density unit.

\begin{tabular}{|l|l|}
\hline Parameter & Value \\
\hline$k_{e r} / \mathrm{ps}^{-1}$ & $8.58 \times 10^{-4} \pm 1 \times 10^{-5}$ \\
\hline$k_{e f} / \mathrm{ps}^{-1} \mathrm{adu}^{-1}$ & $1.14 \pm 2 \times 10^{-2}$ \\
\hline$k_{a} / \mathrm{ps}^{-1} \mathrm{adu}^{-1}$ & $8.84 \times 10^{-3} \pm 1 \times 10^{-4}$ \\
\hline$k_{c} / \mathrm{ps}^{-1}$ & $5.26 \pm 0.16$ \\
\hline$a_{400 \mathrm{~nm} / \mathrm{adu}}$ & $7.47 \pm 0.22$ \\
\hline$a_{510 \mathrm{~nm}} / \mathrm{adu}$ & $9.02 \pm 0.09$ \\
\hline$c_{e x} /-$ & $0.48 \pm 0.02$ \\
\hline$c_{h c} /-$ & $0.57 \pm 0.03$ \\
\hline
\end{tabular}

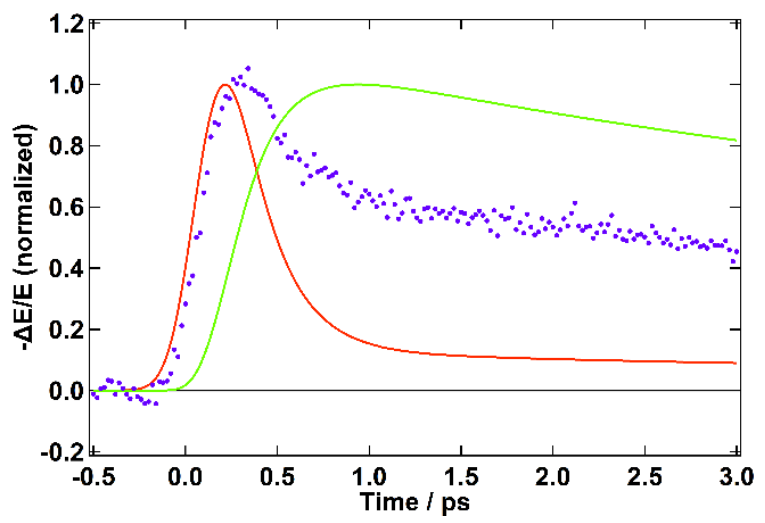

Figure S7. Exciton (green) and carrier (red) evolution according to the global fit on the kinetic model. Excitons show a delayed rise and a certain concentration of carriers is maintained due to Auger heating. 


\section{S4. Influence of the cation}
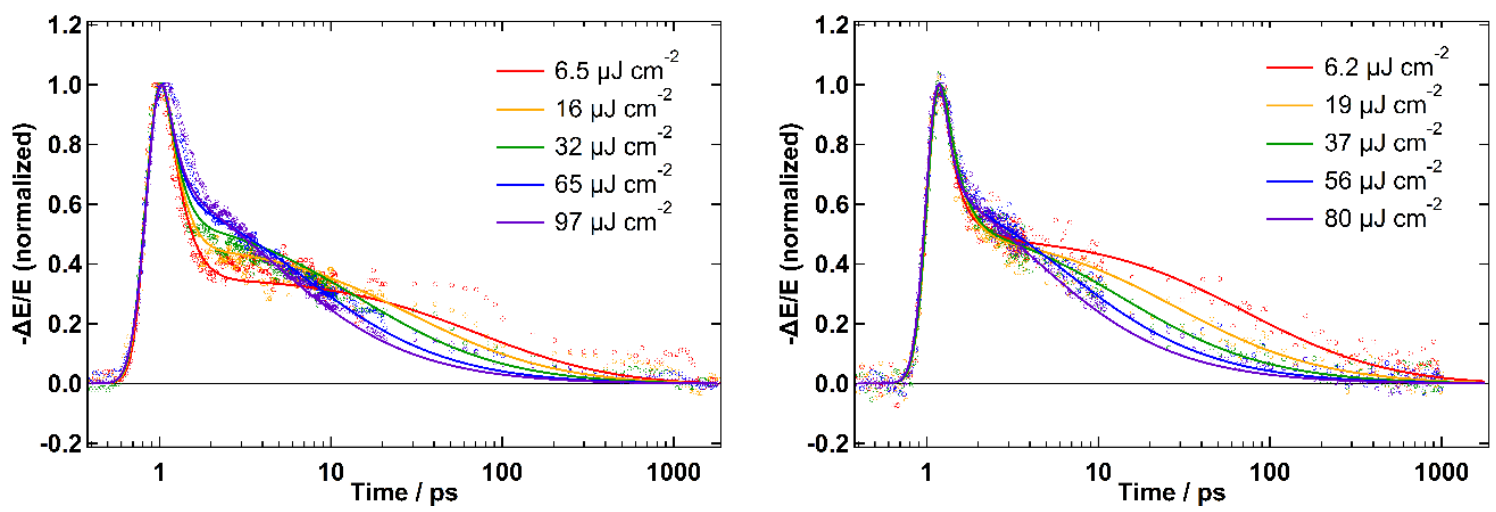

Figure S8. Left) $\mathrm{BuA}_{2} \mathrm{PbI}_{4}$ TRTS dynamics globally fitted using a single value for $k_{c}$. Clearly, there is a deviation between the fit and the data at early times. Right) $\mathrm{PEA}_{2} \mathrm{PbI}_{4}$ TRTS dynamics globally fitted using different values for $k_{c}$. The results do not vary substantially from the results with only one $k_{c}$, unlike $\mathrm{BuA}_{2} \mathrm{PbI}_{4}$ (Figure 4C).

Table S3. Results for the global fit with independent $k_{c}$ for each trace.

\begin{tabular}{|l|l|l|}
\hline & $\mathbf{B u A}_{2} \mathbf{P b I}_{4}$ & $\mathbf{P E A}_{2} \mathbf{P b I}_{4}$ \\
\hline$k_{e r} / \mathrm{ps}^{-1}$ & $1.22 \times 10^{-3} \pm 2 \times 10^{-5}$ & $9.85 \times 10^{-4} \pm 1.6 \times 10^{-5}$ \\
\hline$k_{e f} / \mathrm{ps}^{-1} \mathrm{adu}^{-1}$ & $1.65 \pm 7 \times 10^{-2}$ & $1.68 \pm 5 \times 10^{-2}$ \\
\hline$k_{a} / \mathrm{ps}^{-1} \mathrm{adu}^{-1}$ & $7.10 \times 10^{-3} \pm 1.4 \times 10^{-4}$ & $7.57 \times 10^{-3} \pm 1.3 \times 10^{-4}$ \\
\hline$k_{c 1} / \mathrm{ps}^{-1}$ & $20.5 \pm 2.7$ & $7.44 \pm 7.5 \times 10^{-1}$ \\
\hline$k_{c 2} / \mathrm{ps}^{-1}$ & $5.24 \pm 2.5 \times 10^{-1}$ & $3.82 \pm 1.8 \times 10^{-1}$ \\
\hline$k_{c 3} / \mathrm{ps}^{-1}$ & $3.94 \pm 1.6 \times 10^{-1}$ & $3.66 \pm 1.7 \times 10^{-1}$ \\
\hline$k_{c 4} / \mathrm{ps}^{-1}$ & $1.58 \pm 4 \times 10^{-2}$ & $6.37 \pm 2.5 \times 10^{-1}$ \\
\hline$k_{c 5} / \mathrm{ps}^{-1}$ & $1.22 \pm 3 \times 10^{-2}$ & $6.49 \pm 2.7 \times 10^{-1}$ \\
\hline$a_{400 \mathrm{~nm} / \mathrm{adu}}$ & $4.68 \pm 6 \times 10^{-2}$ & $7.22 \pm 1.4 \times 10^{-1}$ \\
\hline$c_{e x} /-$ & $0.35 \pm 1 \times 10^{-2}$ & $0.56 \pm 3 \times 10^{-2}$ \\
\hline$c_{h c} /-$ & $0.45 \pm 1 \times 10^{-2}$ & $0.78 \pm 4 \times 10^{-2}$ \\
\hline
\end{tabular}



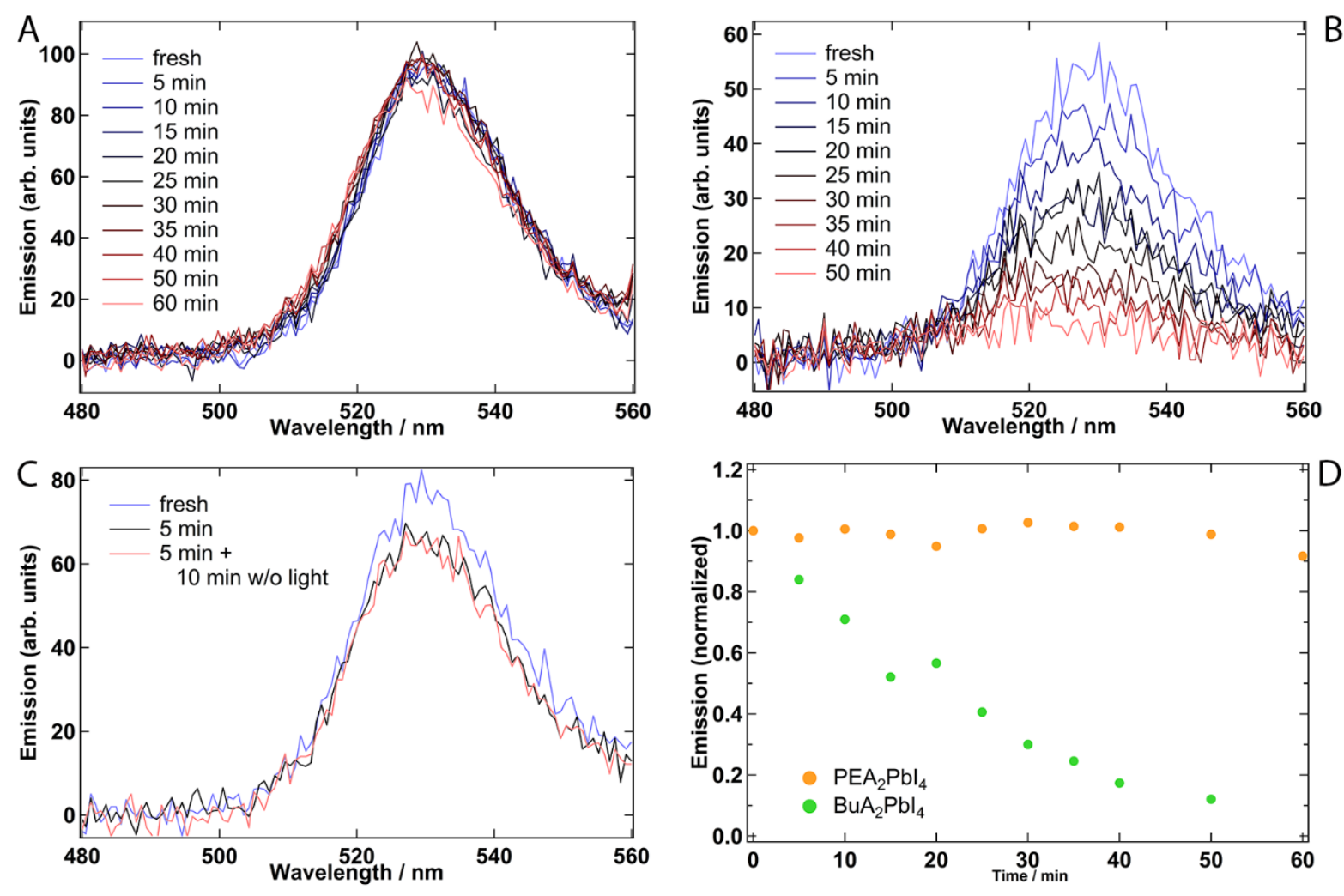

Figure S9. Stability comparison by following the FLUPS emission spectra between $\mathrm{PEA}_{2} \mathrm{PbI}_{4}(\mathrm{~A})$ and $\mathrm{BuA}_{2} \mathrm{PbI}_{4}(\mathrm{~B})$ under laser light illumination. Indeed, $\mathrm{C}$ shows that only under laser light the signal decays. In a few minutes the degradation is substantial for $\mathrm{BuA}_{2} \mathrm{PbI}_{4}$ rendering the FLUPS measurements impossible (D).

\section{S5. Sample Characterization}

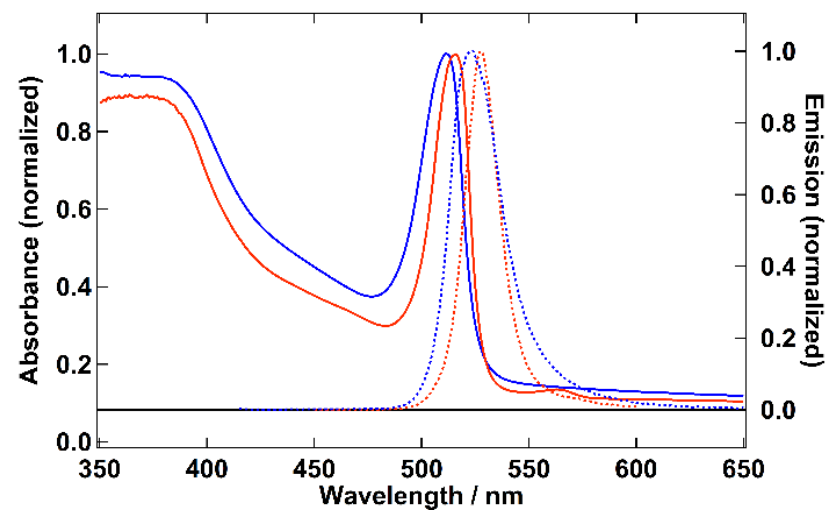

Figure S10. Absorption (solid line) and photoemission (dashed line) spectra of $\mathrm{PEA}_{2} \mathrm{PbI}_{4}$ (red) and $\mathrm{BuA}_{2} \mathrm{PbI}_{4}$ (blue). $\mathrm{BuA}_{2} \mathrm{PbI}_{4}$ shows a small blue-shift and broadening. The latter could be assigned to disorder. ${ }^{4}$ 


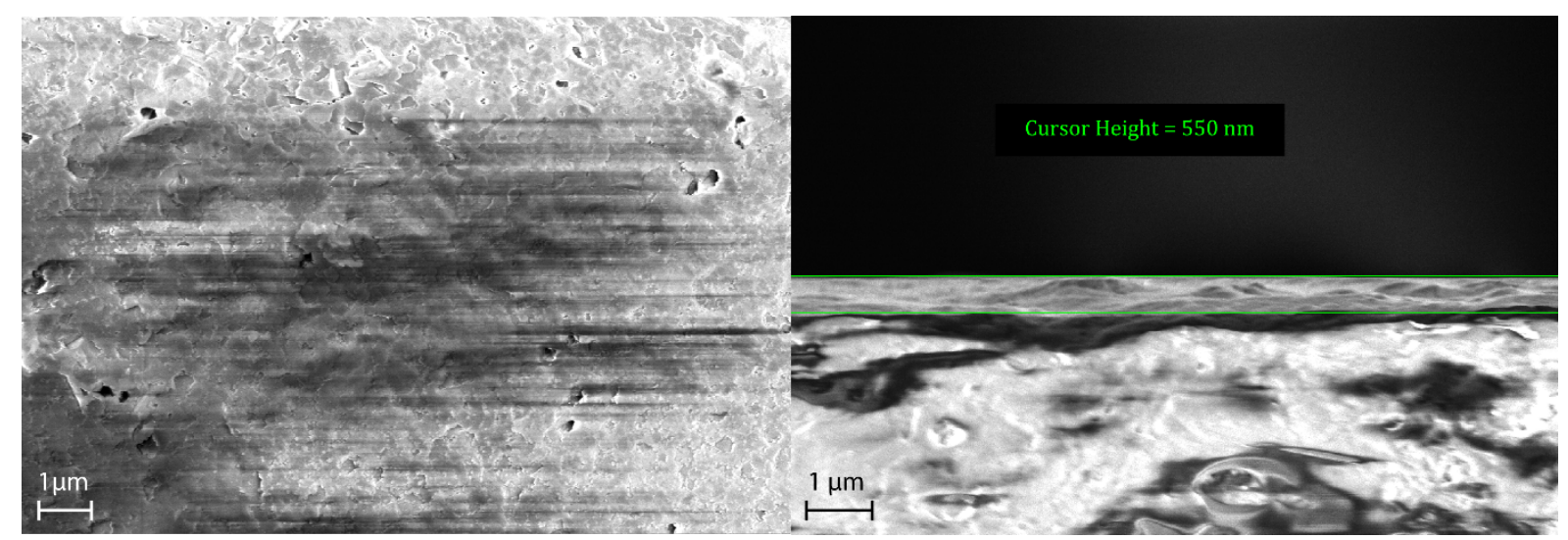

Figure S11. Left) SEM picture of $\mathrm{PEA}_{2} \mathrm{PbI}_{4}$. The flat microscopic crystals appear to lay horizontal to the surface. Right) Cross-sectional SEM showing a thickness of $550 \mathrm{~nm}$ for the $\mathrm{PEA}_{2} \mathrm{PbI}_{4}$ film.

\section{References of the SI}

(1) Nienhuys, H.-K.; Sundström, V. Intrinsic Complications in the Analysis of OpticalPump, Terahertz Probe Experiments. Phys. Rev. B 2005, 71, 235110.

(2) Beard, M. C.; Turner, G. M.; Schmuttenmaer, C. A. Transient Photoconductivity in GaAs as Measured by Time-Resolved Terahertz Spectroscopy. Phys. Rev. B 2000, 62, 15764-15777.

(3) Dexheimer, S. L.; Baxter, J. B.; Schmuttenmaer, C. A. Time-Resolved Terahertz Spectroscopy and Terahertz Emission Spectroscopy, in Terahertz Spectroscopy: Principles and Applications, CRC Press, Boca Raton, 2008.

(4) Ni, L.; Huynh, U.; Cheminal, A.; Thomas, T. H.; Shivanna, R.; Hinrichsen, T. F.; Ahmad, S.; Sadhanala, A.; Rao, A. Real-Time Observation of Exciton-Phonon Coupling Dynamics in Self-Assembled Hybrid Perovskite Quantum Wells. ACS Nano 2017, 11, 10834-10843. 